\section{On the Efficient Implementation of SDA for Boxed Strip-like and Slot-like Structures}

Gabriel Cano, Francisco Medina, and Manuel Horno

\begin{abstract}
This paper reports on an enhanced implementation of the spectral-domain analysis (SDA) of boxed multistrip or multislot transmission lines embedded in a layered medium, including biaxial materials. Very high numerical efficiency is attained by a suitable basis and mixed SDA and spatial-domain technique to calculate the entries of the Galerkin matrix. Convergence properties of SDA are drastically improved, making it competitive with other analytical techniques [such as regular singular integral equation (RSIE)]. The method allows quick and accurate computation of current/field distributions.
\end{abstract}

Index Terms-Analytical methods, planar lines, spectral-domain analysis.

\section{INTRODUCTION}

Spectral-domain analysis (SDA) is considered the standard technique to perform the analysis of planar lines [1], but it is still computationally intensive for computer-aided design (CAD) purposes. Some authors advocate for the use of more analytical methods, such as regular singluar integral equation ([R]SIE) [2], [3]. They claim that (R)SIE is superior to SDA because of a better series convergence and smaller order of characteristic matrices. However, SDA matrices are very small if the proper edge behavior is incorporated into the basis functions. In such a case, these functions have a wide spectrum responsible for poor convergence of spectral series (boxed structures) or integrals (open structures), especially when high spatial resolution (high-order modes, small strips or slots, etc.) is required [4]-[6]. Several methods have been applied to particular structures [7]-[10] so as to improve the convergence. On the other hand, as stated in [3], some conclusions about the efficiency of (R)SIE reported in previous papers were rather optimistic when applied to the analysis of high-order modes or to the accurate description of fields and/or currents. In this paper, we propose an enhanced implementation of SDA overcoming some of its numerical drawbacks. It is suitable for generalized planar structures (strip- and slot-like) embedded in layered iso/anisotropic dielectrics. The key point of the technique is to split the dependence of the spectral series into factors depending on the unknown propagation constant $\gamma$ and the frequency $\omega$ and factors involving the spectral variable sums. Quasi-analytical expressions are provided for these sums. The final result is a very fast code, which makes SDA at least as efficient and accurate as (R)SIE, while retaining its high simplicity.

\section{Statement of The Problem}

The structures under study are boxed planar strip- or slot-like configurations [11, Fig. 1]. Our aim is to compute, as efficiently as possible, the $\gamma$ 's of the supported modes.

Manuscript received October 29, 1997; revised June 29, 1998. This work was supported by the Spanish DGICYT under Project TIC95-0447 and by Watkins-Johnson, under Grant D2010200-14.

G. Cano and F. Medina are with Grupo de Microondas, Departamento de Electronica y Electromagnetismo, Universidad de Sevilla, 41012 Seville, Spain.

M. Horno, deceased, was with Grupo de Microondas, Departamento de Electronica y Electromagnetismo, Universidad de Sevilla, 41012 Seville, Spain.

Publisher Item Identifier S 0018-9480(98)08015-6.

\section{A. Spectral-Domain Formulation}

We start from the linear relationships between fields and currents at the metallized interface (the $M$ th one in [11, Fig. 1]):

1) Slot-Like Structures:

$$
\begin{aligned}
I_{z}(x) & =\int_{-a}^{x} J_{z}\left(x^{\prime}\right) d x^{\prime} \\
E_{z}^{\prime}(x) & =\frac{d E_{z}(x)}{d x} \\
j \omega \mu_{0}\left[\begin{array}{c}
\tilde{J}_{x}(n) \\
\tilde{I}_{z}(n)
\end{array}\right] & =\left[\begin{array}{cc}
\tilde{L}_{x x} & \jmath \frac{\tilde{L}_{x z}}{\alpha_{n}} \\
\jmath \frac{\tilde{L}_{x z}}{\alpha_{n}} & -\frac{\tilde{L}_{z z}}{\alpha_{n}^{2}}
\end{array}\right]\left[\begin{array}{c}
\tilde{E}_{x}(n) \\
\tilde{E}_{z}^{\prime}(n)
\end{array}\right] .
\end{aligned}
$$

2) Strip-Like Structures:

$$
\begin{aligned}
V_{x}(x) & =\int_{-a}^{x} E_{x}\left(x^{\prime}\right) d x^{\prime} \\
J_{x}^{\prime}(x) & =\frac{d J_{x}(x)}{d x} \\
{\left[\begin{array}{c}
\tilde{E}_{z}(n) \\
\tilde{V}_{x}(n)
\end{array}\right] } & =j \omega \mu_{0}\left[\begin{array}{cc}
\tilde{G}_{z z} & \jmath \frac{\tilde{G}_{x z}}{\alpha_{n}} \\
\jmath \frac{\tilde{G}_{x z}}{\alpha_{n}} & -\frac{\tilde{G}_{x x}}{\alpha_{n}^{2}}
\end{array}\right]\left[\begin{array}{c}
\tilde{J}_{z}(n) \\
\tilde{J}_{x}^{\prime}(n)
\end{array}\right]
\end{aligned}
$$

where $J_{\alpha}\left(E_{\alpha}\right)$ are the $\alpha$-components of the surface current density (electric field) at the $M$ th interface. The upper symbol ${ }^{\sim}$ stands for the Fourier transform. $\tilde{G}_{\alpha, \beta}$ and $\tilde{L}_{\alpha, \beta}$ are computed in closed form by using the method in [11]. They are functions of $n, \omega, \gamma$, and the geometrical parameters of the layered configuration.

Equations (2) and (4) have the form $\tilde{\boldsymbol{Y}}(n)=[\tilde{\boldsymbol{F}}(n ; \omega, \gamma)] \tilde{\boldsymbol{X}}(n)$, where $\tilde{\boldsymbol{X}}$ is a source vector and $\tilde{\boldsymbol{Y}}(n)$ is its corresponding field vector. When this relationship is written in the space domain and the boundary conditions imposed by the strips or slots are enforced, we get an integral equation for the source vector. Its spectraldomain kernel is given by $[\tilde{\boldsymbol{F}}(n ; \omega, \gamma)]$. All the components of the unknown source vector in (2) and (4) have the same mathematical behavior. Therefore, we can use a unique set of basis functions to approximate any of the unknown quantities. This provides an elegant unified treatment for strip- and slot-like geometries, and facilitates the analytical preprocessing to be described later. In addition, all the entries of $[\tilde{\boldsymbol{F}}(n, \omega, \gamma)]$ have similar asymptotic behavior for large values of $\alpha_{n}$.

\section{B. Galerkin Method}

The Galerkin method is used to solve our integral equations. Thus, the source vector is expanded into a set of basis functions in such a way that the expansion coefficients $x_{i}=x$ are the solutions of a homogeneous system of linear equations $[\boldsymbol{A}(\omega, \gamma)] \boldsymbol{x}=0$. The solvability condition gives the implicit dispersion equation

$$
\operatorname{det}[\boldsymbol{A}(\omega, \gamma)]=0
$$

For each particular value of $\omega$, there exist nontrivial solutions for an infinite, but discrete, set of values of $\gamma,\left\{\gamma_{n}\right\}$. Solving (5) requires multiple evaluations of $\boldsymbol{A}(\omega, \gamma)$. Therefore, the adequate choice of the basis functions and the method employed to generate $A$ will be crucial to speed up the solution.

\section{Numerical Problems And Their Solutions}

Providing a quick method to generate $\boldsymbol{A}$ (this is our goal) is the key to get high computational speed. A generic element of $\boldsymbol{A}(\omega, \gamma)$ 
has the form of a numerical series involving the Fourier transform of the basis functions $\tilde{\psi}_{\mu}^{i, p}(n)$ and the spectral elements of the Green's dyad $\tilde{F}_{\mu, \nu}(n, \omega, \gamma)$

$$
A_{(i, p)(j, q)}^{\mu, \nu}=\sum_{n}^{\infty} \tilde{\psi}_{\mu}^{i, p}(n) \tilde{F}_{\mu, \nu}(n, \omega, \gamma)\left[\tilde{\psi}_{\nu}^{j, q}(n)\right]^{*}
$$

where $i(j)$ stands for the $i(j)$ th strip or slot, $p(q)$ for the order of the basis function, and $\mu(\nu)$ for the $x$ - or $z$-components. A well-established natural set of basis functions is [9]

$$
\psi_{\mu}^{i, p}(x)=\frac{2}{\pi w_{i}} \frac{T_{i}\left(\frac{x-x_{c i}}{w_{i} / 2}\right)}{\sqrt{1-\left(\frac{x-x_{c i}}{w_{i} / 2}\right)^{2}}}
$$

where $w_{i}$ is the strip (slot) width and $x_{c i}$ the position of its center. Since $\tilde{F}_{\mu, \nu}(n \gg 1) \approx K_{\mu, \nu} / n\left(K_{\mu, \nu}\right.$ being a constant), the general term of (6) diminishes as $n^{-2}$ (very slow rate of convergence) for the basis functions in (7). Although good results can be obtained for $\gamma$ for the first few modes using a reasonable number of Fourier terms $(N)$, this number becomes too large whenever fine spatial resolution is needed. This is a serious drawback for this simple and elegant method. It is then advisable to use ad hoc accelerating techniques (e.g., [7]-[10]). In this paper, we propose a unified and systematic treatment to accelerate computations for very general planar lines and investigate the consequences of its use. The technique is based on the extraction from the original series of auxiliary series in such a way that the difference series converge very fast as follows:

$$
A_{(i, p)(j, q)}^{\mu, \nu}=\sum_{n}^{\infty} \tilde{\psi}_{\mu}^{i, p}(n)\left[\tilde{F}_{\mu, \nu}-\tilde{F}_{\mu, \nu}^{a p}\right]\left[\tilde{\psi}_{\nu}^{j, q}(n)\right]^{*}+S_{(i, p)(j, q)}^{\mu, \nu}
$$

with $\tilde{F}_{\mu, \nu}^{a p}$ being an approximate version of $\tilde{F}_{\mu, \nu}$. The following requisites are demanded from $\left[\tilde{\boldsymbol{F}}^{a p}(n)\right]$.

1) The convergence of the residual series in (8) must be extremely good. Therefore, $\tilde{F}_{\mu, \nu}^{a p}$ should match $\tilde{F}_{\mu, \nu}$ even for low values of $n$ (asymptotic matching must also be enforced).

2) $\tilde{F}_{\mu, \nu}^{a p}$ has to be known in closed form (even for complicated layered structures including biaxial dielectrics).

3) The computation of $S_{(i, p)(j, q)}^{\mu, \nu}$ must be carried out in closed or quasi-closed form.

4) The effort devoted to the evaluation of $S_{(i, p)(j, q)}^{\mu, \nu}$ should be done just once (this is probably the most important condition).

The conditions above are fulfilled by writing the elements of $\tilde{\boldsymbol{F}}^{\text {ap }}$ as the sum of products of functions depending, separately, on $n$ and $(\omega, \gamma)$ as follows:

$$
\tilde{F}_{\mu, \nu}^{a p}(n ; \omega, \gamma)=f_{\mu, \nu}^{(1)}(\omega, \gamma) \tilde{h}_{\mu, \nu}^{(1)}(n)+f_{\mu, \nu}^{(2)}(\omega, \gamma) \tilde{h}_{\mu, \nu}^{(2)}(n)
$$

in such a way that $\tilde{F}_{\mu, \nu}\left(n>n_{0}, \omega, \gamma\right) \approx \tilde{F}_{\mu, \nu}^{a p}(n, \omega, \gamma)$. The integer value $n_{0}$ gives the upper summation limit for the residual series in (8), which have to be repeatedly computed. Therefore, $n_{0}$ should be as small as possible (requirement \#1). Note that the series involving $\tilde{h}_{\mu, \nu}^{(1,2)}$ have to be computed just once for any value of $\gamma$ or $\omega$ (\#4). The functional form of $\tilde{h}_{\mu, \nu}^{(1,2)}$ will be obtained in Section IV for an arbitrary layered structure (\#2). Finally, a very efficient technique to add up the series associated to the approximate Green's dyads is available (Section V and Appendix A) (\#3).

\section{The Approximated Spectral Dyads}

In this section, we obtain $\left[\tilde{\boldsymbol{F}}^{a p}\right]$. Our starting point is the method for the computation of the spectral Green's dyad (SGD) reported in [11]. The elements of the SGD are given there in terms of certain $2 \times$ 2 spectral matrices $[\tilde{\boldsymbol{g}}(n)]_{i, j}$, which are known in closed form. These matrices are written in terms of hyperbolic functions of a certain transverse propagation matrix. In turn, there are three important transverse wavenumbers in that formulation: the eigenvalues of the transverse propagation matrix $\left(\Gamma_{c i}\right.$ and $\left.\Gamma_{\pi i}\right)$ and $\Gamma_{y i}$ [11] (these wavenumbers are identical for isotropic materials, but they differ for anisotropic dielectrics). Let $\Gamma_{i}(n)$ be any of the transverse wavenumbers associated to the $i$ th layer. This generic wavenumber can be expanded as

$$
\Gamma_{i}(n)=a_{1} \alpha_{n}+a_{-1} \alpha_{n}^{-1}+a_{-3} \alpha_{n}^{-3}+\cdots .
$$

In order to build up $\left[\tilde{\boldsymbol{F}}^{a p}(n)\right]$, we truncate (10) as follows:

$$
\Gamma_{i}(n) \approx \Gamma_{i}^{a p}(n)=K_{i} \alpha_{n}+\frac{K_{i}^{\prime 2}(\omega, \gamma)}{2 K_{i}} \frac{k_{0}^{2}}{\alpha_{n}}
$$

where $K_{i}$ and $K_{i}^{\prime}(\omega, \gamma)$ are defined in Appendix B. Some previous asymptotic extraction methods would retain only the first term at the right-hand side in (11). It is easily proven that the integer $n_{0}$, above which $\left|\Gamma_{i}(n)-\Gamma_{i}^{a p}(n)\right|<\delta ; \forall n>n_{0}$ ( $\delta$ is arbitrarily small), increases with $\omega$ as $\omega^{4 / 3}$ for our approximation and as $\omega^{2}$ for the conventional one. Therefore, the advantages of our approach will be more evident when $\omega$ increases. Nevertheless, we only retain the first term in (11) when $\Gamma_{i}(n)$ appears as the argument of a hyperbolic function, i.e.,

$$
\begin{aligned}
\operatorname{coth}\left[\Gamma_{i}(n) h_{i}\right] & \approx \operatorname{coth}\left(K_{i} \alpha_{n} h_{i}\right) \\
\operatorname{cosech}\left[\Gamma_{i}(n) h_{i}\right] & \approx \operatorname{cosech}\left(K_{i} \alpha_{n} h_{i}\right) .
\end{aligned}
$$

This must be done so as to separate the dependence on $(\gamma, \omega)$, such as in (9). Fortunately, the relative error of this approximation of the hyperbolic function is much smaller than the error of the argument itself. The standard large argument approximation for (12) and (13) is 1 and 0 , respectively. This is not a completely convenient choice because the thickness of the dielectric layers is often very small in comparison with the width of the enclosure. Our proposal (12), (13) is advantageous in any case, but especially when the slab-thicknessto-enclosure-width ratio is small.

By applying (11)-(13) in the definitions of $[\tilde{\boldsymbol{g}}(n)]_{i, j}$ in $[11]$, and following the algorithm reported in that paper to compute $[\tilde{\boldsymbol{L}}(n)]$ [a matrix whose elements are $\left.\tilde{L}_{\alpha, \beta}(n)\right]$, we get an approximate version $\left[\tilde{\boldsymbol{L}}^{a p}(n)\right]$ of that dyad as follows:

$$
\left[\tilde{\boldsymbol{L}}^{a p}(n)\right]=\left[\tilde{\boldsymbol{L}}_{A}^{a p}(n)\right]+\frac{1}{2 \alpha_{n}^{2}}\left[\tilde{\boldsymbol{L}}_{B}^{a p}(n)\right]
$$

For strip-like structures, we need an approximation for $[\tilde{\boldsymbol{G}}(n)]$ [a matrix whose entries are $\left.\tilde{G}_{\alpha, \beta}(n)\right]$ with the same features as in (14). This is generated as

$$
\left[\tilde{\boldsymbol{G}}^{a p}(n)\right]=\left[\tilde{\boldsymbol{L}}_{A}^{a p}(n)\right]^{-1}-\frac{1}{2 \alpha_{n}^{2}}\left[\tilde{\boldsymbol{L}}_{A}^{a p}(n)\right]^{-1}\left[\tilde{\boldsymbol{L}}_{B}^{a p}(n)\right]\left[\tilde{\boldsymbol{L}}_{A}^{a p}(n)\right]^{-1}
$$

where the large argument approximation for $\left[\tilde{\boldsymbol{L}}_{A}^{a p}(n)\right]$ has been used. Now using (1)-(4), we can write a unified expression for the approximate version of $[\tilde{\boldsymbol{F}}(n)],\left[\tilde{\boldsymbol{F}}^{a p}(n)\right]$ as follows:

$$
\left[\tilde{\boldsymbol{F}}^{a p}(n ; \omega, \gamma)\right]=\frac{1}{\alpha_{n}}\left\{\left[\tilde{\boldsymbol{F}}_{A}^{a p}(n ; \omega, \gamma)\right]+\frac{k_{0}^{2}}{2 \alpha_{n}^{2}}\left[\boldsymbol{F}_{B}^{a p}(\omega, \gamma)\right]\right\}
$$

where $k_{0}$ is the vacuum wavenumber.

The explicit expressions for the elements of the matrices $\left[\tilde{\boldsymbol{F}}_{A}^{a p}\right]$ and $\left[\tilde{\boldsymbol{F}}_{B}^{a p}\right]$ are as follows. 
A. Case 1: Slot-Like Geometries

$$
\begin{aligned}
\tilde{F}_{A_{x x}}^{a p} & =k_{0}^{2}\left[\tilde{\epsilon}(n)-\frac{\gamma^{2}}{k_{0}^{2}} \tilde{\Phi}(n)\right] \\
\tilde{F}_{A_{x z}}^{a p} & =\tilde{F}_{A_{z x}}^{a p}=\jmath \gamma \tilde{\Phi}(n) \\
\tilde{F}_{A_{z z}}^{a p} & =\tilde{\Phi}(n) \\
\tilde{F}_{B_{x x}}^{a p} & =2 \frac{\gamma^{4}}{k_{0}^{2}}+\gamma^{2}\left(b_{1}-4 b_{2}+b_{3}\right)+k_{0}^{2} b_{4} \\
\tilde{F}_{B_{x z}}^{a p} & =\tilde{F}_{B_{z x}}^{a p}=\jmath \gamma\left(2 b_{2}-b_{3}-2 \frac{\gamma^{2}}{k_{0}^{2}}\right) \\
\tilde{F}_{B_{z z}}^{a p} & =-2 \frac{\gamma^{2}}{k_{0}^{2}}-b_{3} .
\end{aligned}
$$

\section{B. Case 2: Strip-Like Geometries}

$$
\begin{aligned}
\tilde{F}_{A_{z z}}^{a p} & =\frac{\gamma^{2}}{k_{0}^{2} \tilde{\epsilon}(n)}-\frac{1}{\tilde{\Phi}(n)} \\
\tilde{F}_{A_{z x}}^{a p} & =\tilde{F}_{A_{x z}}^{a p}=\jmath \frac{\gamma}{k_{0}^{2} \tilde{\epsilon}(n)} \\
\tilde{F}_{A_{x x}}^{a p} & =-\frac{1}{k_{0}^{2} \tilde{\epsilon}(n)} \\
\tilde{F}_{B_{z z}}^{a p} & =\left(\frac{\gamma}{k_{0} \epsilon}\right)^{2}\left(2 b_{2} \epsilon-b_{4}-\frac{\epsilon^{2}}{2}-\frac{\gamma^{2}}{k_{0}^{2}} b_{1}\right)-\frac{1}{4} b_{3} \\
\tilde{F}_{B_{z x}}^{a p} & =\tilde{F}_{B_{x z}}^{a p}=\jmath \frac{\gamma}{\left(k_{0} \epsilon\right)^{2}}\left(\epsilon b_{2}-b_{4}-\frac{\gamma^{2}}{k_{0}^{2}} b_{1}\right) \\
\tilde{F}_{B_{x x}}^{a p} & =\left(\frac{1}{k_{0} \epsilon}\right)^{2}\left(b_{4}+\frac{\gamma^{2}}{k_{0}^{2}} b_{1}\right)
\end{aligned}
$$

where $\epsilon=\tilde{\epsilon}(n \rightarrow \infty)$. The algorithm to compute $\tilde{\epsilon}(n)$ and $\tilde{\Phi}(n)$, as well as the values of $\epsilon$ and $b_{i}(i=1,2,3,4)$, are given in Appendix B.

Expression (16), in conjunction with (17)-(20), has the form required in (9). Note that the dependence on $\alpha_{n}$ of $\left[\tilde{\boldsymbol{F}}_{A}^{a p}\right]$ and $\left[\tilde{\boldsymbol{F}}_{B}^{a p}\right]$ is quite different: we can consider those terms as first- and second-order approximations to the exact SGD. The details of the stratified dielectric are explicitly incorporated in the first-order term in (16) through $\tilde{\epsilon}(n)$ and $\tilde{\Phi}(n)$. The second-order term in (16) presents a simpler dependence on $n$ and incorporates information about the layers adjacent to the metallized surface. This term becomes important when the ratio $a / \lambda_{0}$ is not small (high frequency). The approximation could be improved while keeping the essential features of our approach by using a more sophisticated expression for $\left[\tilde{\boldsymbol{F}}_{B}^{a p}\right]$. However, this would partially destroy the simplicity of our approach by introducing cumbersome computations, whereas benefits would be significant only for very special structures.

\section{The Asymptotic TAiLs}

Once $\tilde{F}_{\mu, \nu}^{a p}$ is available, direct summation of the numerical series at the right-hand side in (8) is feasible because of their fast convergence. However, the series defining $S_{(i, p)(j, q)}^{\mu, \nu}$ in (8) converge very slowly and their convergence properties are very sensitive to physical dimensions. Even though they have to be computed just once, the computational effort might be prohibitive. It is then necessary to develop a fast method to compute $S_{(i, p)(j, q)}^{\mu, \nu}$. Taking into account the asymptotic limit values of $\tilde{\epsilon}(n)$ and $\tilde{\Phi}(n)$ (see Appendix B), there are two type of numerical series relevant to this task:

$$
S_{t}=\sum_{n=1}^{\infty} \frac{1}{n^{t}} J_{p}\left(n d_{i}\right) J_{q}\left(n d_{j}\right)\left\{\begin{array}{c}
\cos \left(n c_{i}\right) \\
\sin \left(n c_{i}\right)
\end{array}\right\}\left\{\begin{array}{c}
\cos \left(n c_{j}\right) \\
\sin \left(n c_{j}\right)
\end{array}\right\}
$$

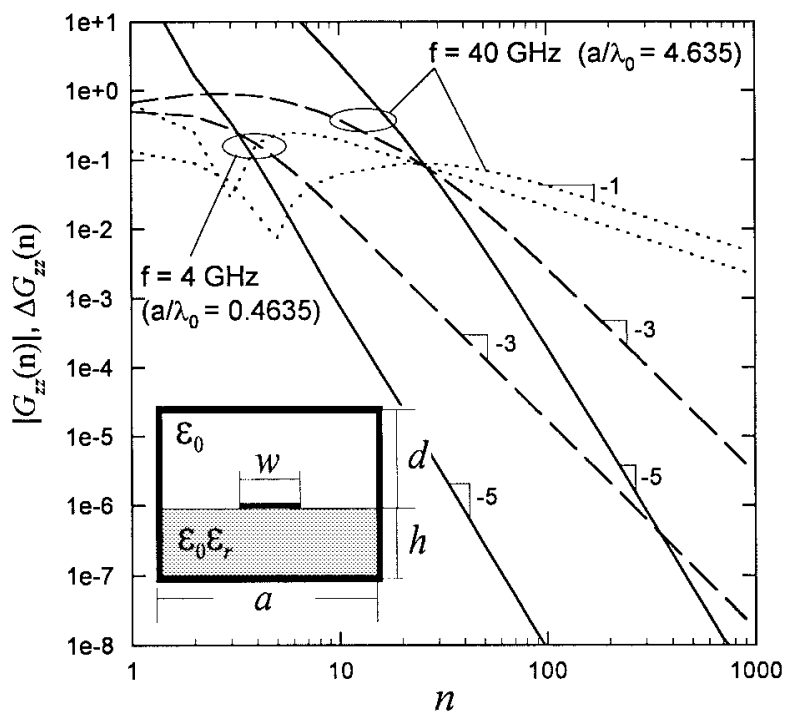

Fig. 1. Dependence on the Fourier variable $(n)$ of $\left|\tilde{G}_{z z}(n)\right|$ (dotted line) and $\Delta \tilde{G}_{z z}=\tilde{G}_{z z}(n)-\tilde{G}_{z z}^{a p}$ (dashed line: first-order approximation, solid line: second-order approximation) for two different frequency values (4 and $40 \mathrm{GHz})$ for a single isotropic substrate in a box $\left(\varepsilon_{r}=11.7\right)$. Dimensions: $h=3.17 \mathrm{~mm}, w=3.04 \mathrm{~mm}, a=34.74 \mathrm{~mm}, d=50 \mathrm{~mm}$.

with $t=1$ and $t=3$. The parameters $d_{i, j}$ and $c_{i, j}$ depend on the strip (slot) widths and their relative locations inside the box. The Bessel functions $J_{p, q}$ multiplied by trigonometric functions in (21) come from the Fourier transforms of (7). The case $t=1$ corresponds to the quasi-static limit of the problem, and quasianalytical expressions have been already provided for this case [12], [13]. The series corresponding to $t=3$ are related to the full-wave nature of the problem, and they become more and more significant when $\omega$ increases. Although we cannot trace for these series a parallel with a quasi-static problem, we can adapt the mathematical procedure underlying one of the methods reported in [12] and [13] to add up such series very efficiently (see Appendix A).

\section{Numerical Results AND Discussion}

In this section, we will check the numerical performance of the techniques described in the previous sections. In particular, we are going to study the following three aspects.

\section{A. Performance of the First- and Second-Order Approximations}

Exact SGD should be compared against its approximations (16). As an example, let us consider the curves in Fig. 1, which correspond to a simple boxed microstrip. The magnitude of the chosen element of the Green's dyad-all the elements behave in a similar way-decreases as $n^{-1}$ for large $n$ (independently of $\omega$ ), while the difference between such an element and their first- and second-order approximations vanishes as $n^{-3}$ and $n^{-5}$. Therefore, the original series decaying as $n^{-2}$ (poor convergence) are substituted by series decaying as $n^{-4}$ or $n^{-6}$ (excellent convergence). This behavior is the same for complex layered structures and for both strip- and slot-like problems. It is clear that extracting out the first-order approximation is almost mandatory to keep the numerical effort within reasonable margins. The secondorder approximation represents an additional improvement of a factor ranging from 5 to 10 (depending on geometry and frequency). Applying asymptotic extraction schemes is particularly important if we are interested in very high-order modes or narrow strips/slots. It is also very important to realize that the singularity of the functions in (7) is eliminated if the series are prematurely truncated (thus, 
TABLE I

Convergence with the Number of Fourier Terms of the Normalized Propagation Constants of the Fundamental $\pi$-Mode and a Complex Mode of Suspended Boxed Coupled Microstrips (See [5] And [6]). $a=2.54 \mathrm{~m}, h_{1}=h_{2}=0.254 \mathrm{~mm}$, $h_{3}=0.762 \mathrm{~mm}, s_{2}=0.0127 \mathrm{~mm}, S_{1}: W_{1}: S_{2}: W_{2}: S_{3}=89.5: 20: 1: 40: 49.5$ $\varepsilon_{r 1}=\varepsilon_{r 3}=1, \varepsilon_{r 2}=2.2, f=150 \mathrm{GHz}$

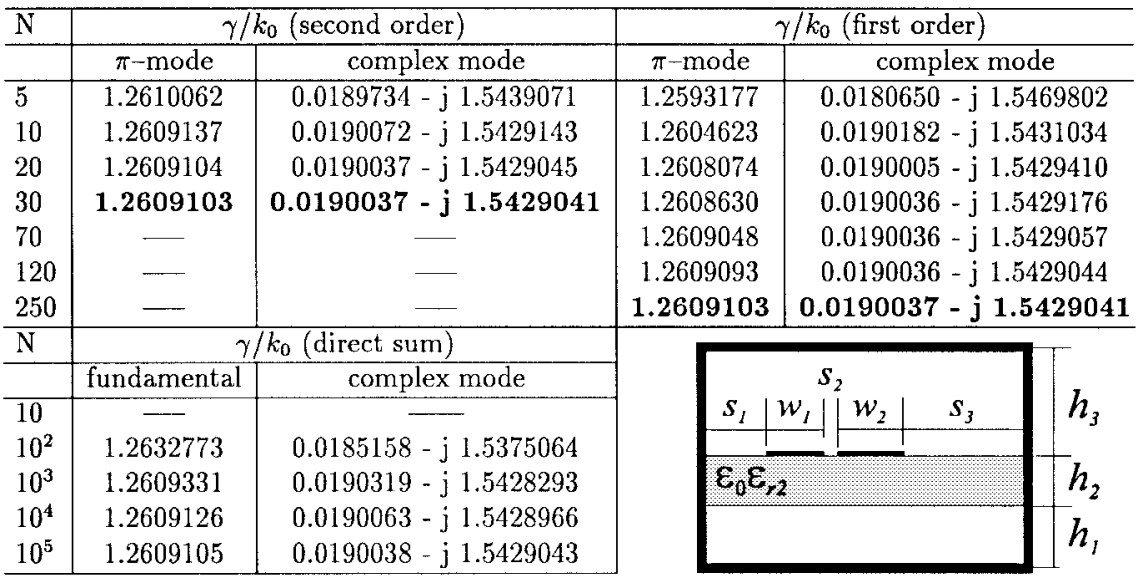

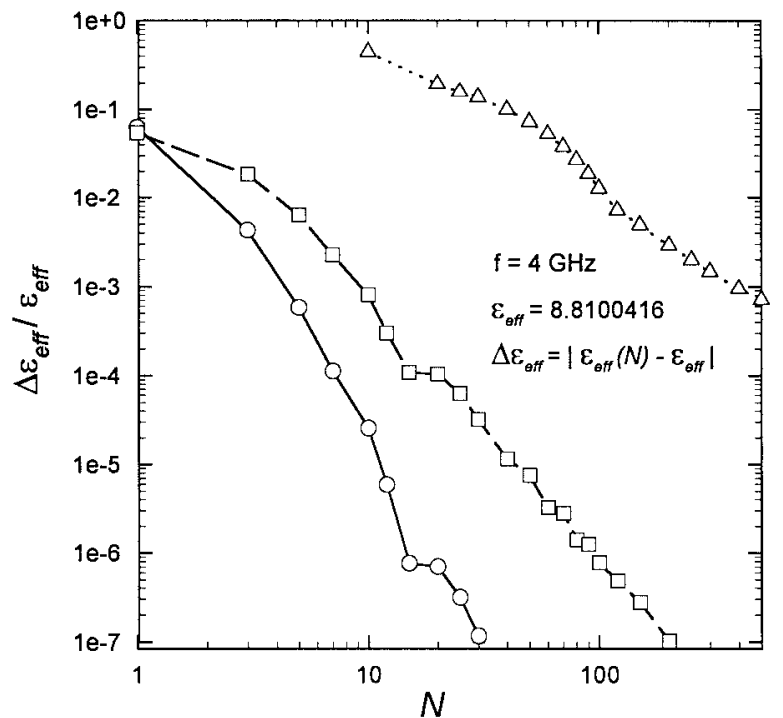

Fig. 2. Relative error of the effective dielectric constant of a boxed microstrip as a function of the number of Fourier terms $(N)$ retained in series evaluations. $\Delta$ : brute-force summation, $\square$ : first-order approximation, $\odot:$ second-order approximation. Physical parameters as in Fig. 1.

losing the nice mathematical features of that basis). The use of asymptotic schemes ensures that the singularity is actually taken into account since it is strongly related to the large argument limit of the spectral series. We have also verified the usefulness of using the approximations in (12) and (13) instead of their limit values of one and zero: when the layer thickness is small, this approach introduces an important improvement.

\section{B. Convergence of the Propagation Constants}

This section illustrates the impact of using our method in the computation of $\gamma$. Let us consider the microstrip of Fig. 1. The curves in Fig. 2 show the error of the fundamental-mode effective dielectric constant as a function of $N$ (eight figures are correct for large $N$ ): asymptotic extraction yields extreme accuracy with few Fourier terms. Second-order approximation is superior to the firstorder one, and both are much better than direct summation. The converged value $\left(\varepsilon_{e f}=8.8100416\right)$ is consistent with the one reported in [8, Table II] (three digits).

In [5] and [6], Tzuang and Kuo presented SDA data for tightly coupled strips in a metallic box, which were computed using thousands of spectral terms. A few terms are enough for our method to get superior accuracy. Thus, Table I reports results for the real $\pi$-mode and the complex mode analyzed in [5] and [6]. Our data systematically and consistently converge to their final values (boldface characters; eight figures are correct) when $N$ increases without oscillations (no numerical instabilities). Convergence rates are dramatically different for direct summation, first-, and second-order approximations. Our results are very close - although not identical - to the ones in [5] and [6]. These convergence patterns are also found in the analysis of multiple conductors or slots embedded in complex stratified regions since the method was designed to account for all those circumstances. The impact of using the technique in this paper is now clear.

\section{Convergence of the Current/Field Expansion}

Obtaining accurate field/current distributions is more difficult than computing good values for $\gamma$. Our method is also suitable for computing those quantities. Returning to the problem analyzed in [5] and [6], we concentrate on the current distribution for the fundamental $\pi$-mode. We have systematically increased the number of basis functions for the surface current on the strips and studied the expansion coefficients. No more than 50 Fourier terms are required to get six or seven correct figures for those (see Table II). We observed that adding a new basis function to the expansion only yields slight variations of the coefficients affecting the other basis functions. This is a consequence of the quasi-orthogonal nature of the functions in (7) for the kernel of our problem. This feature minimizes the number of functions necessary to get a given accuracy. However, taking advantage of this property depends on the accuracy of the evaluation of the Galerkin matrix entries: only the use of asymptotic extraction techniques ensures its proper exploitation, as previously stated. It has also been verified that for low-order modes, the expansion coefficients become smaller and smaller when the order of the affected basis function increases: on a graphical representation of the current distribution, the impact of high-order basis functions is not appreciable, so making the shape of the distribution smooth. In contrast, a nonphysical oscillatory behavior of the computed current density has been reported in [5]. This is attributed to the nature of 
TABLE II

EXPANSION COEFFICIENTS FOR $J_{z}$ ON STRIP \#1 OF THE STRUCTURE IN Table I ( $\pi$-MOde). We Have Used 11 Basis Functions For $J_{z}$ AND TEN FOR $J_{x}$ AT EACH STRIP AND SECOND-ORDER APPROXIMATION

\begin{tabular}{l|c|c|c|c|c}
\hline & $\mathrm{N}=0$ & $\mathrm{~N}=5$ & $\mathrm{~N}=15$ & $\mathrm{~N}=30$ & $\mathrm{~N}=50$ \\
\hline$x_{0}$ & 1.0000000 & 1.0000000 & 1.0000000 & 1.0000000 & 1.0000000 \\
$x_{1}$ & 0.7395056 & 0.7187769 & 0.7193314 & 0.7193278 & 0.7193276 \\
$x_{2}$ & 0.3681362 & 0.3570832 & 0.3573834 & 0.3573817 & 0.3573816 \\
$x_{3}$ & 0.1980832 & 0.1918661 & 0.1919604 & 0.1919658 & 0.1919657 \\
$x_{4}$ & 0.1109432 & 0.1080119 & 0.1080574 & 0.1080592 & 0.1080596 \\
$x_{5}$ & 0.0641157 & 0.0625085 & 0.0625400 & 0.0625386 & 0.0625391 \\
$x_{6}$ & 0.0377435 & 0.0368229 & 0.0368411 & 0.0368406 & 0.0368405 \\
$x_{7}$ & 0.0246870 & 0.0219299 & 0.0219408 & 0.0219409 & 0.0219407 \\
$x_{8}$ & 0.0134350 & 0.0131166 & 0.0131230 & 0.0131231 & 0.0131230 \\
$x_{9}$ & 0.0079645 & 0.0077774 & 0.0077812 & 0.0077812 & 0.0077812 \\
$x_{10}$ & 0.0045061 & 0.0044008 & 0.0044030 & 0.0044030 & 0.0044030 \\
\hline
\end{tabular}

TABLE III

EXPANSION COEFFICIENTS FOR $J_{z}$ ON STRIP \#1 OF THE STRUCTURE IN TABLE I $(\pi$-MODE). WE HAVE USED 11 BASIS FunCTIONS FOR $J_{z}$ AND TEN FOR $J_{x}$ at EACH STRIP AND DiReCt Summation

\begin{tabular}{l|c|c|c|c}
\hline & $\mathrm{N}=100$ & $\mathrm{~N}=600$ & $\mathrm{~N}=2000$ & $\mathrm{~N}=20000$ \\
\hline$x_{0}$ & 1.0000000 & 1.0000000 & 1.0000000 & 1.0000000 \\
$x_{1}$ & 1.5758647 & 0.7344069 & 0.7240906 & 0.7197715 \\
$x_{2}$ & 1.7690514 & 0.3879443 & 0.3663594 & 0.3582137 \\
$x_{3}$ & 1.9751572 & 0.2186382 & 0.2003372 & 0.1927424 \\
$x_{4}$ & 2.6989454 & 0.1590808 & 0.1227672 & 0.1094226 \\
$x_{5}$ & 3.0496396 & 0.1009840 & 0.0745282 & 0.0636590 \\
$x_{6}$ & 4.0618454 & 0.1096000 & 0.0576910 & 0.0387923 \\
$x_{7}$ & 4.1063823 & 0.0735405 & 0.0379886 & 0.0234815 \\
$x_{8}$ & 4.8546689 & 0.1082511 & 0.0404979 & 0.0157688 \\
$x_{9}$ & 3.7514129 & 0.0731321 & 0.0282445 & 0.0099067 \\
$x_{10}$ & 3.6009984 & 0.1211710 & 0.0386809 & 0.0080266 \\
\hline
\end{tabular}

the functions in (7). However, since we do not detect oscillations, we believe that numerical inaccuracy in the summation of the Fourier series (even though thousands of Fourier terms were used) could be the cause of the problem. Tables II and III provide numerical support to this hypothesis. These tables contain the dependence on $N$ of the coefficients for the $z$-directed current expansion on the narrower strip for the $\pi$-mode of the structure in Table I. Table II corresponds to the use of our method and Table III to direct summation. Table II shows excellent convergence. Table III shows the very poor convergence of direct summation $\left(2 \times 10^{4}\right.$ Fourier terms and direct summation yield results, which are much poorer than the ones obtained with $N=0$ and second-order approximation). It is important to emphasize that while the result for $\gamma / k_{0}$ obtained with $N=100$ or 1000 (see Table I) is reasonably good, the current expansion (see Table III) is completely erroneous. Therefore, a truncation of the Fourier series yielding a reasonably good propagation constant may be totally inappropriate for current (or field) distribution computations. The application of our technique completely overcomes this problem. This has been checked with many other examples reported in the literature.

\section{CONCLUSIONS}

This paper presented a procedure to enhance the numerical behavior of the SDA of boxed planar structures. After identifying the drawbacks of the straightforward implementation of SDA, a solution has been proposed based on a technique to speed up spectral series computation. This is based on the use of an appropriate approximation to the Green's dyad, which permits one to reuse cumbersome partial computations. In addition, these partial computations have been carried out in quasi-closed form by using results developed in the context of the quasi-TEM analysis of this type of lines. The formulation has been presented in an unified way for slot- and striplike configurations and it is useful for both propagation constant and field/current computations. The method makes SDA competitive with more analytical techniques.

\section{APPENDIX A}

A fast method to compute the numerical series in (21) is given here. The $t=1$ case in (21) is not considered because it corresponds to the quasi-static limit studied in [12] and [13]. The $t=3$ case still requires further explanation. Expression (21) for $t=3$ actually corresponds to the following series:

$$
S_{3}=\sum_{n=1}^{\infty} \tilde{\psi}_{\mu}^{i, p}(n)\left[\tilde{\psi}_{\nu}^{j, q}\right]^{*} \frac{1}{n^{3}}
$$

where $\tilde{\psi}_{\mu}^{i, p}(n)$ are the Fourier transforms of (7). Parseval and convolution theorems allow us to rewrite (22) as

$$
S_{3}=\frac{a}{2} \int d x \psi_{\mu}^{i, p}(x) \int d x^{\prime} G_{3}\left(x, x^{\prime}\right) \psi_{\nu}^{j, q}\left(x^{\prime}\right)
$$

where

$$
G_{3}\left(x, x^{\prime}\right)=\frac{2}{a} \sum_{n=1}^{\infty} \frac{1}{n^{3}}\left\{\begin{array}{l}
\sin \left(\alpha_{n} x\right) \\
\cos \left(\alpha_{n} x\right)
\end{array}\right\}\left\{\begin{array}{l}
\sin \left(\alpha_{n} x^{\prime}\right) \\
\cos \left(\alpha_{n} x^{\prime}\right)
\end{array}\right\}
$$

where sine (cosine) is used for strip(slot)-like structures. Expression (24) can be written in terms of the function $C N 3(z)=$ $\sum_{n=1}^{\infty} \cos (n z) / n^{3}$. Except for arguments close to 0 or $2 \pi$, there is no closed-form expression for this function, but for such a case there exists an alternative strongly convergent series. In our codes, the following formulas have been used:

\section{A. Small Argument $(z<0.1)$}

$$
\begin{aligned}
\mathrm{CN} 3(z)=1.202056903157+\frac{z^{2}}{2} \ln z & -\frac{3 z^{2}}{4} \\
& -\frac{z^{4}}{288}-\frac{z^{6}}{86,400}-\cdots
\end{aligned}
$$

(this expression can be obviously used when $z$ is close to $2 \pi$ just changing $z$ by $2 \pi-z$ ).

B. Other Case $(0.1<z<2 \pi-0.1)$

$$
\mathrm{CN} 3(z)=S_{1}-\frac{8}{\pi^{2}} S_{2}-\frac{1}{2}\left[\pi z-\frac{z^{2}}{2}-\frac{1+\pi^{2}}{3}\right]
$$

where $S_{1}$ and $S_{2}$ are the following exponentially convergent series,

$$
\begin{aligned}
& S_{1}=\sum_{n=1}^{\infty} \frac{1-\tanh (n)}{n^{3}} \cos (n z) \\
& S_{2}=\sum_{n=1}^{\infty} \frac{\cosh \left[\frac{n \pi}{2}(\pi-z)\right]}{n^{3} \sinh \left(n \pi^{2} / 2\right)} .
\end{aligned}
$$

Due to the regular character of $G_{3}\left(x, x^{\prime}\right)$ and the type of singularities exhibited by (7) at the edges of the integration interval, Gauss-Chebyshev quadratures are specially suitable to perform the double integration in (23). A few quadrature points (no more than the order of the highest order Chebyshev polynomial used in the expansion) typically provide eight or even more exact figures. 


\section{APPENDIX B}

In this appendix, we present the detailed expressions for a number of constants and functions, which are necessary to implement our method. Thus, we provided in (11) an approximation for the $i$ th layer transverse wavenumbers- $\Gamma_{c i}, \Gamma_{\pi i}, \Gamma_{y i}$-relevant to the method used in [11] to calculate the SGD of layered structures with biaxial dielectrics. The parameters $K_{i}$ and $K_{i}^{\prime}$ in (11) are distinct for each transverse wavenumber, being their exact expressions: 1) for $\left.\Gamma_{y i}: K_{i}=1 ; K_{i}^{\prime}=\left(\gamma^{2} / k_{0}^{2}\right)-\varepsilon_{y}^{i} ; 2\right)$ for $\Gamma_{c i}: K_{i}=\sqrt{\varepsilon_{x y}^{i}}$; $K_{i}^{\prime}=\left(\gamma^{2} / k_{0}^{2}\right) \varepsilon_{z y}^{i}-\varepsilon_{x}^{i} ;$ and 3) for $\Gamma_{\pi i}: K_{i}=1 ; K_{i}^{\prime}=\left(\gamma^{2} / k_{0}^{2}\right)-\varepsilon_{z}^{i}$, where $\varepsilon_{x y}^{i}=\varepsilon_{x}^{i} / \varepsilon_{y}^{i}$ and $\varepsilon_{z y}^{i}=\varepsilon_{z}^{i} / \varepsilon_{y}^{i}$.

Let us now write the detailed recurrent algorithm used to calculate $\tilde{\epsilon}(n)$ and $\tilde{\Phi}(n)$ of (17) and (19). Assuming the printed interface is the $M$ th one of a $N$-layers structure, we have (for $1<i<N$ )

$$
\tilde{\epsilon}(n)=\varepsilon_{M+1} \tilde{A}_{M+1}^{+}(n)+\varepsilon_{M} \tilde{A}_{M}^{-}(n)
$$

with

$$
\tilde{A}_{i}{ }^{ \pm}(n)=\frac{\varepsilon_{i}+\varepsilon_{i \pm 1} \tilde{A}_{i \pm 1}^{ \pm}(n) \operatorname{coth}\left[\alpha_{n} \sqrt{\varepsilon_{x y}^{i}} h_{i}\right]}{\varepsilon_{i} \operatorname{coth}\left[\alpha_{n} \sqrt{\varepsilon_{x y}^{i}} h_{i}\right]+\varepsilon_{i \pm 1} \tilde{A}_{i \pm 1}^{ \pm}(n)}
$$

where $\varepsilon_{i}=\sqrt{\varepsilon_{x}^{i} \varepsilon_{y}^{i}}$ and

$$
\tilde{\Phi}(n)=\tilde{\Phi}_{M+1}^{+}(n)+\tilde{\Phi}_{M}^{-}(n)
$$

with

$$
\tilde{\Phi}_{i}^{ \pm}(n)=\frac{1+\tilde{\Phi}_{i \pm 1}^{ \pm}(n) \operatorname{coth}\left(\alpha_{n} h_{i}\right)}{\operatorname{coth}\left(\alpha_{n} h_{i}\right)+\tilde{\Phi}_{i \pm 1}^{ \pm}(n)} .
$$

This recurrent algorithm is initialized with the following values:

$$
\begin{aligned}
\tilde{A}_{1}^{-} & =\operatorname{coth}\left[\alpha_{n} \sqrt{\varepsilon_{x y}^{1}} h_{1}\right] \\
\tilde{A}_{N}^{+}(n) & =\operatorname{coth}\left[\alpha_{n} \sqrt{\varepsilon_{x y}^{N}} h_{N}\right] \\
\tilde{\Phi}_{1}^{-}(n) & =\operatorname{coth}\left(\alpha_{n} h_{1}\right) \\
\tilde{\Phi}_{N}^{+}(n) & =\operatorname{coth}\left(\alpha_{n} h_{N}\right) .
\end{aligned}
$$

Note that the limit values for large $n$ of $\tilde{\epsilon}(n)$ and $\tilde{\Phi}(n)$ are $\epsilon=\varepsilon_{M+1}+\varepsilon_{M}$ and 2 , respectively.

The $b_{i}(i=1,2,3,4)$ in (18) and (20) are expressed in terms of the electrical parameters of the $M$ th and $M+1$ th layers

$$
\begin{aligned}
b_{1}= & \varepsilon_{M} \frac{\varepsilon_{z}^{M}}{\varepsilon_{x}^{M}}+\varepsilon_{M+1} \frac{\varepsilon_{z}^{M+1}}{\varepsilon_{x}^{M+1}} \\
b_{2}= & \varepsilon_{M} \frac{\varepsilon_{z}^{M}-\varepsilon_{y}^{M}}{\varepsilon_{x}^{M}-\varepsilon_{y}^{M}}+\varepsilon_{y}^{M} \frac{\varepsilon_{x}^{M}-\varepsilon_{z}^{M}}{\varepsilon_{x}^{M}-\varepsilon_{y}^{M}} \\
& +\varepsilon_{M+1} \frac{\varepsilon_{z}^{M+1}-\varepsilon_{y}^{M+1}}{\varepsilon_{x}^{M+1}-\varepsilon_{y}^{M+1}}+\varepsilon_{y}^{M+1} \frac{\varepsilon_{x}^{M+1}-\varepsilon_{z}^{M+1}}{\varepsilon_{x}^{M+1}-\varepsilon_{y}^{M+1}} \\
b_{3}= & \varepsilon_{z}^{M}+\varepsilon_{z}^{M+1} b_{4}=\varepsilon_{y}^{M} \varepsilon_{M}+\varepsilon_{y}^{M+1} \varepsilon_{M+1} .
\end{aligned}
$$

\section{REFERENCES}

[1] D. Mirshekar-Syahkal, Spectral Domain Method for Microwave Integrated Circuits. Norwood, MA: Artech House, 1990.

[2] Y.-F. Huang and S.-L. Lai, "Regular solution of shielded planar transmission lines," IEEE Trans. Microwave Theory Tech., vol. 42, pp. 84-91, Jan. 1994.

[3] Y.-S. Xu and A. S. Omar, "Rigorous solution of mode spectra for shielded multilayer microstrip lines," IEEE Trans. Microwave Theory Tech., vol. 42, pp. 1213-1222, July 1994.

[4] S. El-Gazhaly and W. J. R. Hoefer, "Effect of number of spectral terms on convergence of spectral-domain calculations of $E$-plane structures," Electron. Lett., vol. 21, no. 16, pp. 677-678, Aug. 1985.

[5] C.-K. C. Tzuang and J.-T. Kuo, "Modal current distributions on closely coupled microstrip lines: A comparative study of the SDA basis functions," Electron. Lett., vol. 26, no. 7, pp. 464-465, Mar. 1990.

[6] J. T. Kuo and C. C. Tzuang, "Complex modes in shielded suspended coupled microstrip lines," IEEE Trans. Microwave Theory Tech., vol. 38, pp. 1278-1286, Sept. 1990.

[7] C. J. Railton and J. P. McGeehan, "A rigorous and computationally efficient analysis of microstrip for use as an electro-optic modulator," IEEE Trans. Microwave Theory Tech., vol. 37, pp. 1099-1104, July 1989.

[8] G. Coen, N. Fache, and D. De Zutter, "Comparison between two sets of basis functions for the current modeling in the Galerkin spectral domain solution for microstrips," IEEE Trans. Microwave Theory Tech., vol. 42, pp. 505-513, Mar. 1994.

[9] T. Rozzi, F. Moglie, A. Morini, E. Marionna, and M. Politi, "Hybrid modes, substrate leakage, and losses of slotline at millimeterwave frequencies," IEEE Trans. Microwave Theory Tech., vol. 38, pp. 1069-1078, Aug. 1990.

[10] G. Cano, F. Medina, and M. Horno, "Efficient spectral domain analysis of generalized multistrip lines in stratified media including thin, anisotropic and lossy substrates," IEEE Trans. Microwave Theory Tech., vol. 40, pp. 217-227, Feb. 1992.

[11] F. Medina, M. Horno, and H. Baudrand, "Generalizad spectral analysis of planar lines on layered media including uniaxial and biaxial dielectric substrates," IEEE Trans. Microwave Theory Tech., vol. 37, pp. 504-511, Mar. 1989.

[12] E. Drake, F. Medina, and M. Horno, "Improved quasi-TEM spectral domain analysis of boxed coplanar multiconductor microstrip lines," IEEE Trans. Microwave Theory Tech., vol. 41, pp. 260-267, Feb. 1993.

[13] _ "Quick computation of $[C]$ and $[L]$ matrices of generalized multiconductor coplanar waveguide transmission lines," IEEE Trans. Microwave Theory Tech., vol. 42, pp. 2328-2335, Dec. 1994.

\section{ACKNOWLEDGMENT}

The authors wish to thank Dr. D. Swanson, Watkins \& Johnson, Palo Alto, CA, for his motivation and material support. 